\title{
On Hypermomentum in General Relativity II. The Geometry of Spacetime
}

\author{
Friedrich W. Hehl, G. David Kerlick* , and Paul von der Heyde \\ Institut für Theoretische Physik der Universität zu Köln
}

(Z. Naturforsch. 31 a, 524-527 [1976] ; received March 29, 1976)

\begin{abstract}
In Part $\mathrm{I}^{* *}$ of this series we have introduced the new notion of hypermomentum $\Delta i j k$ as a dynamical quantity characterizing classical matter fields. In Part II, as a preparation for a general relativistic field theory, we look for a geometry of spacetime which will allow for the accomodation of hypermomentum into general relativity. A general linearly connected spacetime with a metric $\left(\mathrm{L}_{4}, g\right)$ is shown to be the appropriate geometrical framework.
\end{abstract}

\section{Einstein's General Relativity and $U_{4}$ Theory}

The success of relativistic quantum field theory in describing fundamental processes continues to affirm the validity of Minkowski's flat spacetime $R_{4}$ (notation of Schouten ${ }^{1}$ ) as a model of reality, at least where gravitational fields are not so strong as to dominate.

Einstein adopted a spacetime for general relativity (GR) which retains locally, in the neighborhood of a point, the characteristics of Minkowski spacetime. This Riemannian spacetime $\mathrm{V}_{4}$ derives all of its properties from the metric tensor $g_{i j}(i, j \ldots=0,1$, 2,3 ; see Part I for notation). Interpreted physically, the metric tensor represents infinitesimal spacetime intervals as measured by standard clocks and rods (for a detailed description see Misner, Thorne, and Wheeler ${ }^{2}$, Ch. 13).

Levi-Civita offered an additional interpretation of the Riemannian space in terms of parallel transport of vectors. In addition to the metric structure, a $\mathrm{V}_{4}$ has an affine structure as well. The Christoffel symbol

$$
\left\{\begin{array}{l}
k \\
i j
\end{array}\right\}:=g^{k l} \Delta_{j i l}^{a b c}\left[\frac{1}{2} \partial_{a} g_{b c}\right]
$$

is the affine connection in a $\mathrm{V}_{4}$. Here, the permutation tensor $\Delta_{j i l}^{a b c}$ has been defined by

$$
\unlhd_{j i l}^{a b c}:=\delta_{j}^{a} \delta_{i}^{b} \delta_{l}^{c}+\delta_{i}^{a} \delta_{l}^{b} \delta_{j}^{c}-\delta_{l}^{a} \delta_{j}^{b} \delta_{i}^{c} .
$$

Because of the relationship (1), the affine properties of a $V_{4}$ can be expressed in terms of the metric tensor. There is no independent affine structure in a $\mathrm{V}_{4}$; everything is subordinate to the metric ("no affine freedom"). The extremal curves (shortest or

\footnotetext{
* Alexander von Humboldt fellow.
}

** Z. Naturforsch. 31 a, 111 [1976].

Reprint requests to Prof. Dr. F. W. Hehl, Institut für Theoretische Physik der Universität zu Köln, Zülpicher Straße 77, D-5000 Köln 41. longest lines with respect to the metric) coincide with autoparallel curves (straightest lines with respect to the connection). The Christoffel connection in GR can be interpreted physically as a "guiding field" because the motion of structureless nonrotating test particles (which can be derived from the field equations) turns out to be autoparallel with respect to the connection.

After the appearance of GR, mathematicians stimulated by Einstein's work developed a plethora of new geometries, among which the Riemannian $\mathrm{V}_{4}$ is a special case (compare Schouten ${ }^{1}$ and Schrödinger ${ }^{3}$ ). In the last few years, the most general affine space compatible with a Riemannian metric (the Riemann-Cartan space $\mathrm{U}_{4}$ ) was successfully applied to an extension of Einstein's GR. In the gravitational theory of that spacetime, spin angular momentum is coupled to torsion just as energy-momentum is coupled to the metric. We take this extended gravitational theory, the so-called $\mathrm{U}_{4}$ theory, for granted and refer to the literature ${ }^{4-6}$ for the available evidence.

Motivated by the considerations on hypermomentum in Part I, we will investigate a still more general geometry, namely the general affine geometry $\mathrm{L}_{4}$ with metric $g$. In this framework, which we denote by $\left(\mathrm{L}_{4}, g\right)$, the metric and the affine structures are decoupled, as are their physical interpretations.

\section{The General Affine Space with Metric $\left(\mathrm{L}_{4}, \mathrm{~g}\right)$}

Allow the 64 components of the affine connection $\Gamma_{i j}^{k}$ to be completely arbitrary, instead of being given by Equation (1). Then prescribe the (symmetric) metric tensor field $g_{i j}$ of signature +2 (ten independent components) and let these two fields $\left(\Gamma_{i j}^{k}, g_{i j}\right)$ be completely unrelated. What structures can we extract from these assumptions? 
Without using the metric, we can split the connection $\Gamma_{i j}^{k}$ into its symmetric and its antisymmetric part. The symmetric part $\Gamma_{(i j)}^{k}$ is still an affinity, transforming inhomogeniously under general coordinate transformations. The antisymmetric part $S_{i j}{ }_{i j}^{k}:=\Gamma_{[i j]}^{i k}$ transforms as a tensor and has 24 independent components (Cartan's torsion). The torsion measures the non-commutativity of covariant derivatives on scalar fields (and the closure failure of infinitesimal parallelograms).

The geometrical importance of the metric $g_{i j}$ suggests that its covariant derivative $Q_{i j k}:=-\nabla_{i} g_{j k}$ (40 independent components) should be an important quantity in an $\left(\mathrm{L}_{4}, g\right)$. This tensor, which we shall call nonmetricity, measures the failure of the connection to preserve the metric during parallel transport. The vanishing of $Q_{i j k}$ in an $\left(\mathrm{L}_{4}, g\right)$ is the metric condition which defines a $\mathrm{U}_{4}$ geometry. In the corresponding $U_{4}$ theory ${ }^{4-6}$, we regard the vanishing of $Q_{i j k}$ as a reflection of physical reality: on a macroscopic scale, there exist global length (time) and angle standards which can be related by parallel displacement. In the neighborhood of each point, spacetime is Euclidean with respect to metric and (anholonomic) connection ${ }^{7}$.

It will be useful to split the nonmetricity into a trace $Q_{i}$ and a traceless part $\bar{Q}_{i j k}$ :

$$
Q_{i j k}=Q_{i} g_{j k}+\bar{Q}_{i j k} \text {. }
$$

Here, $Q_{i}:=Q_{i l}^{l l} / 4$ and $\bar{Q}_{\ddot{i} l}^{l} \equiv 0$.

With these definitions of torsion and nonmetricity at hand, we can express the connection in terms of $g, Q$, and $S$ by means of the following identity:

$$
\begin{aligned}
\Gamma_{i j}^{k} & \equiv g^{k l} \Delta_{j i l}^{a b c}\left[\frac{1}{2} \partial_{a} g_{b c}-g_{c d} S_{\ddot{a} b}^{. d}+\frac{1}{2} Q_{a} g_{b c}+\frac{1}{2} \bar{Q}_{a b c}\right] \\
& =\left\{\begin{array}{l}
k \\
i j
\end{array}\right\}+W_{i j}^{. k}=\left\{\begin{array}{l}
k \\
i j
\end{array}\right\}-M_{i j}{ }_{i j}^{k}+\frac{1}{2} Q_{i j} . k
\end{aligned}
$$

The definitions of $W_{i j}^{k}$ and of the generalized contortion tensor $M_{i j k}=-W_{i[j k]}$ in terms of $S_{i j}^{k}$ and $Q_{i j k}$ can be read off from (3) and (1). In the case where $Q_{i j k}=0$, the generalized contortion reduces to the contortion of $\mathrm{U}_{4}$ theory.

By means of (3), we have separated the affinity and the tensor part of the connection. The affinity part $\left\{\begin{array}{l}k \\ i j\end{array}\right\}$ is expressed in terms of the metric tensor. So it is a matter of convenience which of the following alternatives we use for specifying the $\left(\mathrm{L}_{4}, g\right)$ :

$$
\begin{array}{cc} 
& \left(\Gamma_{i j}^{k}, g_{i j}\right), \quad\left(g_{i j}, S_{i j}^{k}, Q_{i j k}\right) \quad(4 \mathrm{a}, \mathrm{b}, \mathrm{c}) \\
\text { or } \quad\left(g_{i j}, M_{i j k}, Q_{i j k}\right) & \text { etc. }
\end{array}
$$

In contrast to $(4 \mathrm{a})$, the sets $(4 \mathrm{~b}, \mathrm{c})$ comprise only tensor fields. In each case we can prescribe 74 in- dependent fields. Therefore $(4 \mathrm{a}),(4 \mathrm{~b})$, and $(4 \mathrm{c})$ are equivalent for the description of the $\left(\mathrm{L}_{4}, g\right)$. The set Equation (4c) will turn out to be particularly convenient: the generalized contortion $M_{i j k}$ couples to the spin of matter, whereas the nonmetricity $Q_{i j k}$ measures the deviations from $\mathrm{U}_{4}$ theory. Let us now analyze the affine connection of an $\left(\mathrm{L}_{4}, g\right)$ by means of a moving orthonormal tetrad.

\section{The Moving Tetrad}

The presence of a hyperbolic metric on an $\mathrm{X}_{4}$ allows us to define locally at each point an (anholonomic) orthonormal tetrad (frame) of vectors $\boldsymbol{e}_{\mu}$ which satisfy $\boldsymbol{e}_{\mu} \cdot \boldsymbol{e}_{\nu}=g_{\mu \nu}=\operatorname{diag}(-1,+1,+1$, $+1)$ at every point $(\mu, v \ldots=0,1,2,3)$. When the $\mathrm{X}_{4}$ is an $\mathrm{L}_{4}$, the parallel transport of tetrads provides a suggestive visualization of the properties of the connection $\Gamma_{i j}^{k}$ in Equation (3). We follow here essentially the presentation of Kröner ${ }^{8}$.

The Christoffel part of the connection in tetrad coordinates (the well-known Ricci rotation coefficients), causes only a rotation of the frames during parallel transport with respect to the given tetrad system. The tensor $M_{i j k}=-M_{i k j}$ causes an additional, independent rotation of the frame. Thus the tensor $M_{i j k}$ suggests itself as the appropriate variable for defining dynamical spin in an $\left(\mathrm{L}_{4}, g\right)$.

The vector part of the nonmetricity (better known as the Weyl vector) causes a uniform change of scale in a parallelly transported tetrad. Relative lengths of the tetrad vectors and angles between them remain unaltered. Such a geometrical variable suggests itself as a counterpart to the dilatation current.

The traceless part of the nonmetricity $\bar{Q}_{i j}^{k}$ causes a shear of the tetrad vectors which does not preserve angles or relative lengths, but which does preserve the volume $\operatorname{det}\left(\boldsymbol{e}_{\mu}\right)$ of the 4-parallelepiped whose sides are given by the tetrad vectors. The dynamical counterpart that we expect to arise here is the traceless proper hypermomentum defined in Part I. We express our results symbolically:

$$
\begin{array}{rr}
\text { tetrad connection of the }\left(\mathrm{L}_{4}, g\right) & (16 \times 4) \\
\sim \text { contortion } & (6 \times 4) \\
\oplus \text { (relative) dilatation } & (1 \times 4) \\
\oplus \text { (relative) shear } & (9 \times 4)
\end{array}
$$

\footnotetext{
* Instead of "relative rotation", we use the more convenient
} term "contortion". 
The term relative should remind us that these deformations compare two tetrads at different points of an $\left(\mathrm{L}_{4}, g\right)$. In Part III the tetrad deformations of Eq. (5) will be related to the corresponding dynamical quantities in Eq. (8) of Part I.

In the following section we will investigate the conditions imposed on a geometrical framework by the requirement that it leads to a consistent physical interpretation. To facilitate this discussion, we classify the specializations of the spacetime manifold $\left(\mathrm{L}_{4}, g\right)$ as follows (see also Figure 1$)$ :

The vanishing of $\bar{Q}_{i j k}$ specializes the $\left(\mathrm{L}_{4}, g\right)$ to a Weyl spacetime with torsion $\mathrm{Y}_{4}$.

The vanishing of $Q_{i j k}$ (i.e. of $\bar{Q}_{i j k}$ and $Q_{i}$ ) defines a Riemann-Cartan spacetime $\mathrm{U}_{4}$.

The vanishing of $\bar{Q}_{i j k}$ and $S_{i j}^{k}$ defines a Weyl spacetime $\mathrm{W}_{4}$.

The vanishing of $Q_{i j k}$ and $S_{i j}^{k}$ (and thus of $W_{i j} i^{k}$ ) reduces spacetime to the Riemannian spacetime $\mathrm{V}_{4}$. Should $\left\{{ }_{i j}^{k}\right\}$ then also vanish globally, the spacetime would be the Minkowski $\mathrm{R}_{4}$.

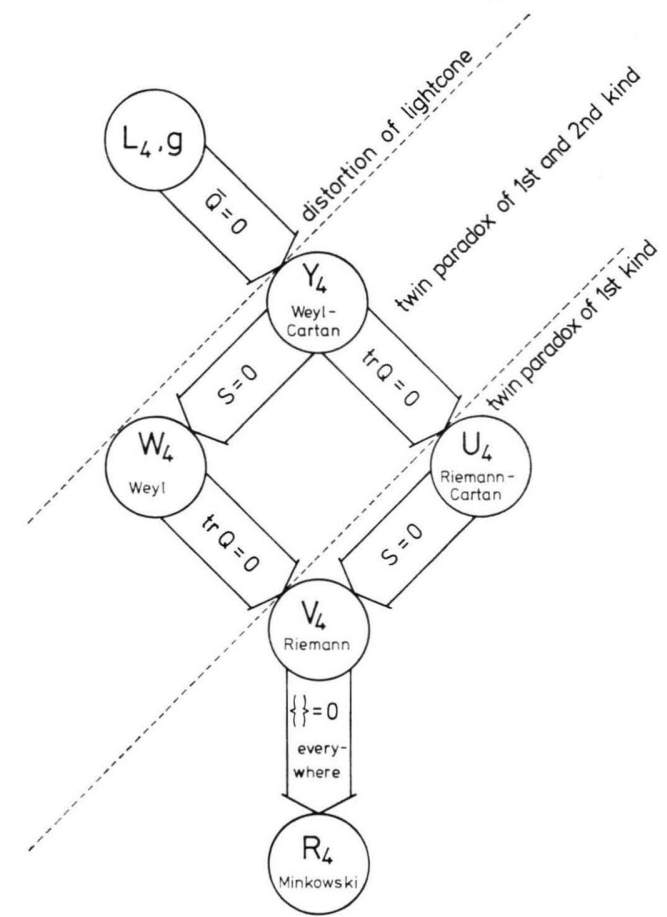

Fig. 1. Classification of $\left(\mathrm{L}_{4}, g\right)$ geometries according to the connection of Equation (3) .

\section{On Projective and Conformal Concepts; the Geometry of the Physical World}

Important for making the affine connection physical is the axiomatic point of view propounded by
Ehlers, Pirani, and Schild (EPS) ${ }^{9}$. In this approach, the primitive concepts from which the geometry of spacetime is to be deduced are the paths of light rays and of structureless, massive, non-rotating test particles. The paths of light rays determine a conformal structure (light cone at each point) on a differentiable manifold. The paths of massive particles determine a set of autoparallel curves (a projective structure). EPS discovered that these structures taken together already determine the differentiable and affine structure of spacetime. When torsion is excluded, the most general geometrical frame. work compatible with the conformal and the projective structure is a $W_{4}$. Quite obviously, if torsion is permitted one has a $\mathrm{Y}_{4}$.

Apart from these restrictions, EPS require a new hypothesis: There shall be no "twin paradox of the second kind." That is, two observers bearing identical clocks and rods shall not find, meeting each other after travelling via different spacetime paths, that their size scales are different and that their clocks tick at different rates. When no such effect is present, the geometry is found to simplify from a $\mathrm{W}_{4}$ to $\mathrm{V}_{4}$. The corresponding reduction, when torsion is not eliminated by assumption, is from a $\mathrm{Y}_{4}$ to a $\mathrm{U}_{4}$.

Start once more with the manifold $\left(\mathrm{L}_{4}, g\right)$. The connection and metric together comprise 64 plus 10 variables. In macroscopic physics, where special relativity is valid locally, we observe no "twin paradox of the second kind", so we take the restriction $Q_{i j k}=0$ (40 equations) to be a valid hypothesis here. The $\mathrm{U}_{4}$ geometry of the world has 34 degrees of freedom.

Suppose, however, that the "second twin paradox" can occur in the microscopic realm. Then the four components $Q_{i}$ would no longer be fixed, but would be determinable from some matter current (from the dilatation current, we expect). Perhaps then the $\mathrm{Y}_{4}$ geometry (38 independent components) is a better picture for high energies. That this geometry occurs in the local gauge theory of the Weyl group, has already been shown by Bregman ${ }^{10}$ and others.

If, in addition, we allow a nonvanishing $\bar{Q}_{i j k}$, all 74 components of the affine geometry would be determined by matter in the microscopic realm. When we picture the effects of a $\bar{Q}_{i j k}$ on geometry in terms of rods and clocks, a number of very unpleasant consequences come to light. The light cone 
is no longer invariant under parallel transport; it can be stretched and tipped; spacelike vectors (rods) after parallel transport become timelike vectors (clocks), and so on. Clearly this sort of behavior cannot be allowed for physical rods and clocks, and for observations in macroscopic physics. However, thought experiments with rods and clocks make little sense in the domain of elementary particles. The onset of quantum phenomena in this range makes the concept of a well defined light cone of zero thickness harder to justify (consider, for example, the problems with "light cone singularities" in quantum field theory). Similarly, the validity of a local Lorentz structure at these distances is hard to prove. Therefore we hold open the question of whether $\bar{Q}$ terms could be allowed in nature. The formalism, including the field equations, which emerge will be mathematically consistent in any case, and will embody all the simplest transformations (linear ones) of vectors in spacetime.

We sum up in a second working hypothesis: spacetime possesses a general affine structure and a

1 J. A. Schouten, Ricci Calculus, 2nd ed., Springer-Verlag, Berlin 1954.

2 C. W. Misner, K. S. Thorne, and J. A. Wheeler, Gravitation, Freeman, San Francisco 1973.

3 E. Schrödinger, Space-time Structure, reprinted with corr., Cambridge Univ. Press 1960.

4 A. Trautman, Ann. N. Y. Acad. Sci. 262, 241 [1975].

5 F. W. Hehl, P. von der Heyde, and G. D. Kerlick, Phys. Rev. D 10, 1066 [1974]. metric structure $\left(\mathrm{L}_{4}, g\right)$, which allow an orthonormal tetrad to undergo all possible linear transformations during parallel transport. Such a geometry should provide the basis for dynamical definitions of the Hilbert type. We expect the macroscopic world to be adequately described by $\mathrm{U}_{4}$ geometry ("no second twin paradox in the large") or by a $\mathrm{Y}_{4}$ geometry in the scaling limit. That these specializations of an $\left(\mathrm{L}_{4}, g\right)$ are adequate to describe physics "in the large", should be seen to result from certain macroscopic properties of matter. We do not restrict a priori the $\left(\mathrm{L}_{4}, g\right)$ geometry in the microscopic world.

We are grateful to Professor Peter Mittelstaedt and members of his relativity group, and to Professor Ekkehart Kröner and Dr. Karl-Heinz Anthony for interesting discussions. Furthermore, we thank Professor Jürgen Ehlers for a discussion on the EPS axiomatics. One of us (G.D.K.) would like to thank the Humboldt Foundation for the award of a fellowship.

6 F. W. Hehl, P. von der Heyde, G. D. Kerlick, and J. M. Nester, Rev. Mod. Phys., July 1976 (in press).

7 P. von der Heyde, Lettr. Nuovo Cim. 14, 250 [1975].

8 E. Kröner, in A. Sommerfeld "Vorlesungen über theoretische Physik", Vol. 2, Chap. 9; 5th ed., Akad. Verlagsges., Leipzig 1964.

9 J. Ehlers, F. A. E. Pirani, and A. Schild, Festschrift for Synge (L. O'Raifeartaigh ed.), Oxford Univ. Press 1972.

10 A. Bregman, Progr. Theor. Phys. 49, 667 [1973]. 